\title{
Composición Corporal y Somatotipo en Futbol Femenino. Campeonato Sudamericano Sub-17
}

\author{
Body Composition and Somatotype in Women's Football. South American Championship Sub-17 \\ "Carlos Bahamondes Avila; **Bernarda Macarena Cifuentes Cea; \\ ${ }^{* * * *}$ Eleazar Lara Padilla \& ${ }^{* * * *}$ Francisco José Berral de la Rosa
}

BAHAMONDES, A. C.; CIFUENTES, C. B. M.; LARA, P. E. \& BERRAL, R. F. J. Composición corporal y somatotipo en futbol femenino. Campeonato sudamericano sub-17. Int. J. Morphol., 30(2):450-460, 2012.

RESUMEN: Con el objetivo de analizar la composición corporal y el somatotipo en seleccionadas nacionales de futbol femenino, se han estudiado 28 mujeres, 19 seleccionadas de Colombia y 9 de Paraguay, de 16,2 \pm 0,66 años de edad, participantes en el Campeonato Sudamericano Sub 17 - Chile 2008, clasificatorio para la primera Copa Mundial FIFA de la categoría, Nueva Zelanda 2008. Para la evaluación se siguió el protocolo establecido por la Sociedad Internacional para el Avance de la Cineantropometria - ISAK. Se determinó el fraccionamiento del peso corporal total en cinco masas según Kerr. Para establecer el somatotipo se utilizó el modelo propuesto por Heath \& Carter. Respecto a las masas corporales, el promedio de los kg y \% de MA, MM y MO fue de 18,56 kg-33,28\%; $21,80 \mathrm{~kg}-39,31 \% ; 6,20 \mathrm{~kg}-11,18 \%$. La clasificación del somatotipo es de endomorfo-mesomorfo, en el cual hay una dominancia equilibrada de adiposidad y muscularidad sobre la linealidad relativa. Los SDD del SM indican que no existen diferencias significativas $(\mathrm{p}<0,05)$ entre los diferentes puestos de juego, y el SDI sugiere que el grupo total de evaluadas es homogéneo.

PALABRAS CLAVE: Fútbol; Antropometría; Rendimiento muscular; Índice músculo-óseo; Biotipo.

\section{INTRODUCCIÓN}

La configuración morfológica de un deportista puede ser estudiada mediante su composición corporal y somatotipo o biotipo, que permiten describir y comparar distintos niveles de rendimiento. En deportes colectivos, sobre todo en la etapa de especialización, donde la selección de talentos se hace compleja y multifactorial, facilita la selección deportiva y la elección del puesto de juego. Diversos autores han planteado que aún cuando la selección de talentos sea un procedimiento impreciso, el conocimiento del perfil de salud de jugadores exitosos ha sido un recurso valioso para identificar talentos y por consiguiente, seleccionarlo y entrenarlo (Mujika et al., 2009).

La valoración de la composición corporal y el somatotipo en el deporte puede aportar información relevante respecto a las dimensiones corporales de las jugadoras de elite (Reilly et al., 2000), dado que las características físicas son consideradas un requisito importante en el desempeño atlético (Sharma \& Dixit, 1985). Por ello, deter- minar la morfología corporal a partir de las variables antropométricas es parte de la valoración rutinaria de cualquier deportista, permitiendo ubicar a este y compararlo en un deporte o puesto de juego, de tal forma que permita mejorar el rendimiento individual y/o colectivo o plantear regímenes de entrenamiento con la intención de encuadrar a los atletas en la condición más próxima a una referencia (Queiroga et al., 2008). Así, cuantificar las masas plásticas, adiposa y muscular, facilita cuantificar el peso extra o de lastre y su relación con la eficiencia o rendimiento mecánico, ayuda a interpretar mejor los efectos anabólicos del entrenamiento físico o ejercicio, su relación con el coste metabólico, la producción de fuerza, la capacidad de trabajo físico y el rendimiento muscular (Berral et al., 2010).

Dado todo lo anterior, y considerando que la información respecto a las características morfológicas en jugadoras de fútbol es escasa, principalmente en Sudamérica, el objetivo del estudio es determinar la composición corporal

\footnotetext{
* Magíster en Medicina y Ciencias del Deporte. Profesor Asociado Universidad Mayor, Temuco, Chile.

** Licenciada en Kinesiología. Docente Universidad Santo Tomás, Valdivia. Universidad Mayor, Temuco, Chile.

**** Doctor en Ciencias. Profesor Titular y Director del Área de Postgrado del Instituto Politécnico Nacional de México (IPN), México DF. México.

***** Doctor en Medicina y Cirugía. Profesor Titular y Director del Doctorado en Alto Rendimiento Deportivo, Universidad Pablo de Olavide (UPO). Sevilla, España.
} 
y el somatotipo en seleccionadas nacionales de futbol femenino participantes en el Campeonato Sudamericano Sub 17 - Chile 2008, clasificatorio a la primera Copa Mundial FIFA de la categoría, Nueva Zelanda 2008.

\section{MATERIAL Y METODO}

Participaron del estudio 28 mujeres, 19 seleccionadas de Colombia y 9 de Paraguay, de 16,2 \pm 0,66 años de edad; ambos equipos fueron los finalistas (campeón y subcampeón) del torneo Sudamericano de fútbol femenino, categoría Sub 17, realizado en Chile, durante enero de 2008. La evaluación se realizó el día previo a la final del torneo, teniendo en cuenta la declaración de Helsinky, respecto a los principios éticos de investigación humana, donde cada deportista participó de forma voluntaria, firmando para ello un consentimiento informado, contando además con la autorización del cuerpo técnico y médico. Para la evaluación se siguió el protocolo establecido por la Sociedad Internacional para el Avance de la Cineantropometria - ISAK (Marfell-Jones et al., 2006), con ello se determinó la composición corporal por el método de Kerr (1988) el cual fracciona el peso corporal total en cinco masas: muscular, adiposa, ósea, residual y piel. Para establecer el somatotipo se utilizó el modelo propuesto por Heath \& Carter (Heath \& Carter, 1967; Carter \& Heath, 1990) y la clasificación corresponde a la traducción literal del trabajo original. Las 44 variables medidas las realizaron antropometristas certificados por la ISAK como Nivel II, utilizando para ello un estadiómetro calibrado con cinta metálica Stanley y con una precisión de $1 \mathrm{~mm}$; una balanza clínica marca Detecto, con una precisión de 100 gr, un plicómetro marca Harpenden con una precisión de $0,2 \mathrm{~mm}$, dos antropómetros, tipo Campbell, de brazos cortos y largos, y una cinta antropométrica, todos marca Roscraft, modelo Gaucho Pro, y con una precisión de $1 \mathrm{~mm}$.

Con lo anterior, se determinaron de forma individual las variables de interés: peso $(\mathrm{P})$, estatura (E), estatura sentada (ES), índice de masa corporal (IMC), suma de seis pliegues $\left(\sum 6 \mathrm{Pl}\right)$, masas corporales $(\%-\mathrm{Kg})$ : muscular $(\mathrm{MM})$, adiposa (MA), ósea (MO), residual (MR) y piel (MP), índice músculo óseo (IMO) y somatotipo, con sus respectivas coordenadas y su clasificación biotipológica o cualitativa (Carter, 2000), para luego agrupar a las deportistas por puesto de juego en: Arqueras (ARQ) - Defensas (DEF) - Medios Defensivos (MDE) - Medios Ofensivos (MOF) - Delanteras (DEL).

Para el análisis descriptivo se registraron el promedio y desviación estándar por puesto de juego, utilizando la prueba de Kolmogorov-Smirnov para determinar la distri- bución normal y para comparar los diferentes grupos el test de Kruskal - Wallis; además se incorporó el análisis bidimensional, sistema propio del somatotipo, el cual contempla la distancia de dispersión del somatotipo medio o SDD del SM, comparando bidimensionalmente un grupo con una referencia, y el índice de dispersión del somatotipo o SDI, determinando la homogeneidad grupal o dispersión del somatotipo de un grupo en relación a su somatotipo medio (Heath \& Carter; Carter \& Heath; Esparza Ros, 1993; Carter).

\section{RESULTADOS}

En la Tabla I se muestran las características antropométricas de la muestra. Se puede observar que las ARQ poseen un mayor P, E, ES, E6PL e IMC. Respecto a las masas corporales, el promedio de los $\mathrm{kg}$ y $\%$ de MA, MM y MO fue de $18,56 \mathrm{~kg}-33,28 \% ; 21,80 \mathrm{~kg}-39,31 \% ; 6,20$ $\mathrm{kg}-11,18 \%$, respectivamente, donde nuevamente las ARQ destacan con un mayor desarrollo en kg de MM y MA, no así en \%, donde la MM es mayor en DEF y la MA es mayor en MOF. Sobre el IMO es mayor en las ARQ y menor en las MOF, esto principalmente dado por un mayor desarrollo de los $\mathrm{Kg} \mathrm{MM}$, no así en los kg MO, donde se mantiene dentro del rango del total del grupo, incluso en las primeras se observa que está casi medio punto por encima del promedio. En el somatotipo (Fig. 1), la clasificación del grupo es de endomorfo-mesomorfo, en el cual hay un equilibrio de adiposidad y muscularidad sobre la linealidad relativa. Los SDD del SM indican que no existen diferencias significativas $(\mathrm{p}<0,05)$ entre los diferentes puestos de juego, y el SDI sugiere que el grupo total de evaluadas es homogéneo.

Para el análisis estadístico se incluyeron a las 28 jugadoras de futbol agrupadas por puesto de juego, con excepción de las 4 arqueras, en las demás posiciones de juego se integraron 6 casos por cada posición.

En general, de acuerdo al estadístico de Kolmogorov Smirnov, la forma de distribución de todas las variables antropométricas medidas fue semejante a la normal. Una vez analizadas el conjunto de variables, según posición de juego (Tablas II, III, IV), sólo en dos de ellas se observó una tendencia a una diferencia de interés, caracterizándose porque en las arqueras prevaleció un menor porcentaje de Masa Ósea $(\mathrm{p}=$ $0,08)$ junto con un mayor Índice Musculo-esquelético $(\mathrm{p}=$ $0,14)$. Dicha tendencia se manifiesta por los valores de $\mathrm{p}$, que solo están cercanos a la significancia estadística, lo cual puede indicar la presencia del error tipo beta, es decir, la significancia podría existir, pero no se manifiesta por el tamaño pequeño de la muestra, lo cual es soportado por la significancia estadística del estudio calculada post-hoc $(68,4 \%)$. 
Fig 1. Somacarta con los somatotipos medios por puestos de juego.
Tabla I. Características Antropométricas Evaluadas por posición de juego y total: Edad, peso (P), estatura (E), estatura sentada (ES), sumatoria de seis pliegues ( $\mathrm{C} 6 \mathrm{PL})$, índice de masa corporal (IMC), masas corporales cinco componentes de Kerr (kg-\%), masa grasa dos componentes de Durning \& Womersley (\%MG), índice músculo óseo (IMO), somatotipo medio, clasificación biotipológica, distancia de dispersión del somatotipo medio (SDD del SM) e índice de dispersión del somatotipo (SDI).

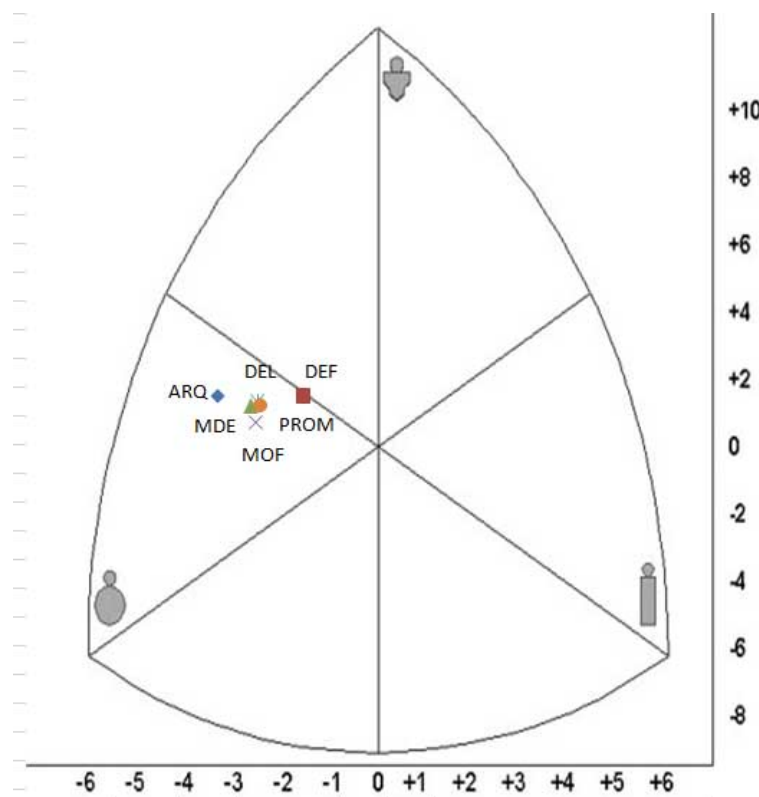

\begin{tabular}{|c|c|c|c|c|c|c|}
\hline & ARQ & DEF & MDE & MOF & DEL & Total \\
\hline n & 4 & 6 & 6 & 6 & 6 & 28 \\
\hline Edad & $16,22 \pm 0,40$ & $16,37 \pm 0,72$ & $16,21 \pm 0,77$ & $16,33 \pm 0,94$ & $16,08 \pm 0,45$ & $16,25 \pm 0,66$ \\
\hline $\mathbf{P}$ & $60,50 \pm 4,21$ & $55,02 \pm 3,45$ & $56,28 \pm 5,37$ & $55,68 \pm 5,70$ & $57,30 \pm 3,97$ & $56,70 \pm 4,64$ \\
\hline $\mathbf{E}$ & $162,08 \pm 4,33$ & $160,53 \pm 3,36$ & $159,62 \pm 3,19$ & $160,13 \pm 3,14$ & $160,13 \pm 5,13$ & $160,39 \pm 3,66$ \\
\hline ES & $89,38 \pm 1,84$ & $86,87 \pm 1,25$ & $88,18 \pm 3,02$ & $87,57 \pm 3,19$ & $87,72 \pm 3,21$ & $87,84 \pm 2,60$ \\
\hline$\sum 6 \mathrm{PL}$ & $101,30 \pm 25,80$ & $81,70 \pm 8,94$ & $90,45 \pm 19,27$ & $94,13 \pm 24,02$ & $88,58 \pm 22,54$ & $90,51 \pm 19,92$ \\
\hline IMC & $23,01 \pm 0,43$ & $21,35 \pm 1,22$ & $22,07 \pm 1,70$ & $21,72 \pm 2,15$ & $22,40 \pm 2,16$ & $22,05 \pm 1,69$ \\
\hline Kg MA & $20,54 \pm 0,17$ & $17,28 \pm 1,34$ & $18,42 \pm 0,17$ & $19,05 \pm 0,17$ & $18,16 \pm 0,13$ & $18,56 \pm 0,15$ \\
\hline Kg MM & $24,13 \pm 3,04$ & $21,66 \pm 1,31$ & $21,58 \pm 3,42$ & $20,58 \pm 3,73$ & $21,85 \pm 3,11$ & $21,80 \pm 2,99$ \\
\hline Kg MO & $6,14 \pm 0,62$ & $6,14 \pm 0,52$ & $6,29 \pm 0,28$ & $6,27 \pm 0,63$ & $6,13 \pm 0,57$ & $6,20 \pm 0,54$ \\
\hline Kg MR & $5,98 \pm 2,85$ & $5,64 \pm 0,53$ & $5,86 \pm 2,40$ & $5,34 \pm 2,47$ & $5,53 \pm 1,55$ & $5,65 \pm 2,24$ \\
\hline Kg MP & $3,44 \pm 0,58$ & $3,28 \pm 0,12$ & $3,30 \pm 0,66$ & $3,29 \pm 0,96$ & $3,33 \pm 0,65$ & $3,32 \pm 0,65$ \\
\hline IMO & $3,94 \pm 0,51$ & $3,55 \pm 0,35$ & $3,44 \pm 0,23$ & $3,31 \pm 0,34$ & $3,58 \pm 0,25$ & $3,50 \pm 0,36$ \\
\hline \%МА & $34,09 \pm 0,04$ & $31,97 \pm 0,01$ & $33,03 \pm 0,03$ & $34,73 \pm 0,03$ & $32,86 \pm 0,03$ & $33,28 \pm 0,03$ \\
\hline$\% \mathrm{MM}$ & $40,04 \pm 0,04$ & $40,12 \pm 0,02$ & $38,95 \pm 0,02$ & $37,87 \pm 0,03$ & $39,85 \pm 0,02$ & $39,31 \pm 0,02$ \\
\hline$\% \mathrm{MO}$ & $10,23 \pm 0,01$ & $11,38 \pm 0,01$ & $11,37 \pm 0,01$ & $11,47 \pm 0,01$ & $11,15 \pm 0,00$ & $11,18 \pm 0,01$ \\
\hline$\% \mathrm{MR}$ & $9,92 \pm 0,01$ & $10,46 \pm 0,01$ & $10,68 \pm 0,01$ & $9,85 \pm 0,01$ & $10,06 \pm 0,00$ & $10,21 \pm 0,01$ \\
\hline$\% \mathrm{MP}$ & $5,73 \pm 0,00$ & $6,08 \pm 0,00$ & $5,98 \pm 0,00$ & $6,08 \pm 0,01$ & $6,09 \pm 0,00$ & $6,01 \pm 0,00$ \\
\hline$\% \mathrm{MG}$ & $30,88 \pm 4,52$ & $27,71 \pm 2,19$ & $29,43 \pm 3,02$ & $29,90 \pm 4,39$ & $28,40 \pm 3,17$ & $29,15 \pm 3,38$ \\
\hline ENDO & $4,63 \pm 1,41$ & $3,72 \pm 0,48$ & $4,31 \pm 0,91$ & $4,44 \pm 1,18$ & $4,19 \pm 1,32$ & $4,23 \pm 1,00$ \\
\hline MESO & $3,92 \pm 0,23$ & $3,79 \pm 0,38$ & $3,74 \pm 0,66$ & $3,68 \pm 1,03$ & $3,75 \pm 0,88$ & $3,77 \pm 0,70$ \\
\hline ЕСТO & $1,66 \pm 0,19$ & $2,34 \pm 0,67$ & $1,95 \pm 0,81$ & $2,18 \pm 1,11$ & $1,96 \pm 1,05$ & $2,04 \pm 0,80$ \\
\hline $\mathbf{X}$ & $-3,00$ & $-1,40$ & $-2,40$ & $-2,30$ & $-2,20$ & $-2,20$ \\
\hline $\mathbf{Y}$ & 1,50 & 1,50 & 1,20 & 0,70 & 1,40 & 1,30 \\
\hline Clasificación & Meso- & Mesomorfa- & Meso- & Meso- & Mesomorfa- & Mesomorfa- \\
\hline Biotipo & Endomorfa & Endomorfa & Endomorfa & Endomorfa & Endomorfo & Endomorfa \\
\hline SDD del SM & 1,40 & 1,40 & 0,30 & 0,50 & 0,10 & SDI 0,80 \\
\hline
\end{tabular}


Tabla II. Análisis estadístico comparativo por posición de juego: medidas de base.

\begin{tabular}{|c|c|c|c|c|c|}
\hline \multirow[t]{6}{*}{ Edad } & Arquera & 4 & 16,2218 & 0,40364 & \multirow{6}{*}{0,956} \\
\hline & Defensa & 6 & 16,3746 & 0,71571 & \\
\hline & Delantera & 6 & 16,0794 & 0,44793 & \\
\hline & Medio defensiva & 6 & 16,2122 & 0,77267 & \\
\hline & Medio ofensiva & 6 & 16,3340 & 0,93843 & \\
\hline & Total & 28 & 16,2460 & 0,66005 & \\
\hline \multirow[t]{6}{*}{ Peso } & Arquera & 4 & 60,5000 & 4,20951 & \multirow{6}{*}{0,442} \\
\hline & Defensa & 6 & 55,0167 & 3,45336 & \\
\hline & Delantera & 6 & 57,3000 & 3,97341 & \\
\hline & Medio defensiva & 6 & 56,2833 & 5,36970 & \\
\hline & Medio ofensiva & 6 & 55,6833 & 5,70067 & \\
\hline & Total & 28 & 56,7036 & 4,64499 & \\
\hline \multirow[t]{6}{*}{ Talla } & Arquera & 4 & 162,0750 & 4,33465 & \multirow{6}{*}{0,898} \\
\hline & Defensa & 6 & 160,5333 & 3,36254 & \\
\hline & Delantera & 6 & 160,1333 & 5,13290 & \\
\hline & Medio defensiva & 6 & 159,6167 & 3,19400 & \\
\hline & Medio ofensiva & 6 & 160,1333 & 3,14113 & \\
\hline & Total & 28 & 160,3857 & 3,65571 & \\
\hline \multirow[t]{6}{*}{ Talla sentada } & Arquera & 4 & 89,3750 & 1,83916 & \multirow{6}{*}{0,690} \\
\hline & Defensa & 6 & 86,8667 & 1,25167 & \\
\hline & Delantera & 6 & 87,7167 & 3,21087 & \\
\hline & Medio defensiva & 6 & 88,1833 & 3,02352 & \\
\hline & Medio ofensiva & 6 & 87,5667 & 3,18915 & \\
\hline & Total & 28 & 87,8393 & 2,59948 & \\
\hline \multirow[t]{6}{*}{ Suma 6 pliegues } & Arquera & 4 & 101,3000 & 25,80362 & \multirow{6}{*}{0,657} \\
\hline & Defensa & 6 & 81,7000 & 8,94136 & \\
\hline & Delantera & 6 & 88,5833 & 22,54262 & \\
\hline & Medio defensiva & 6 & 90,4500 & 19,27483 & \\
\hline & Medio ofen siva & 6 & 94,1333 & 24,02155 & \\
\hline & Total & 28 & 90,5143 & 19,91956 & \\
\hline \multirow[t]{6}{*}{ Indice de Masa Corporal } & Arquera & 4 & 23,0067 & 0,43101 & \multirow{6}{*}{0,621} \\
\hline & Defensa & 6 & 21,3501 & 1,21798 & \\
\hline & Delantera & 6 & 22,4036 & 2,16363 & \\
\hline & Medio defensiva & 6 & 22,0713 & 1,69912 & \\
\hline & Medio ofensiva & 6 & 21,7167 & 2,15298 & \\
\hline & Total & 28 & 22,0456 & 1,68887 & \\
\hline
\end{tabular}

\section{DISCUSIÓN}

Las características antropométricas generales de las jugadoras de fútbol evaluadas son menores en peso y estatura que una muestra de 15 jugadoras brasileñas de fútbol sala de edades similares, con registros de $62,47 \pm 8,38 \mathrm{~kg}$ de peso y $165,0 \pm 0,7 \mathrm{~cm}$ de estatura (Levandosky et al., 2007). Situación dispar ocurre con una muestra de 17 jugadoras "top-class" turcas de 20,73 $\pm 2,09$ años, donde su peso era de $56,63 \pm 5,03 \mathrm{~kg}$ y su estatura de $162,4 \pm 5,79 \mathrm{~cm}$ (Can et 
BAHAMONDES, A. C.; CIFUENTES, C. B. M.; LARA, P. E. \& BERRAL, R. F. J. Composición corporal y somatotipo en futbol femenino. Campeonato sudamericano sub-17. Int. J. Morphol., 30(2):450-460, 2012.

Tabla III. Análisis estadístico comparativo por posición de juego: \% de masas fraccionadas.

\section{\% Masa muscular}

\% Masa ósea

$\%$ Masa residual

\% Masa piel

Indice Músculo óseo

\% Masa grasa (Durning \& Womersley)

\begin{tabular}{|c|c|c|c|c|}
\hline & $\mathbf{n}$ & Media & Desviación típica & $\mathbf{P}$ \\
\hline Arquera & 4 & 34,0925 & 4,39791 & \multirow[t]{6}{*}{0,570} \\
\hline Defensa & 6 & 31,9650 & 1,37078 & \\
\hline Delantera & 6 & 32,8567 & 2,75450 & \\
\hline Medio defensiva & 6 & 33,0267 & 2,93192 & \\
\hline Medio ofensiva & 6 & 34,7267 & 3,45164 & \\
\hline Total & 28 & 33,2793 & 2,94982 & \\
\hline Arquera & 4 & 40,0350 & 3,58321 & \multirow[t]{8}{*}{0,473} \\
\hline Defensa & 6 & 40,1167 & 1,73588 & \\
\hline Delantera & 6 & 39,8467 & 2,28647 & \\
\hline Medio defensiva & 6 & 38,9450 & 1,51571 & \\
\hline Medio ofensiva & 6 & 37,8667 & 2,87501 & \\
\hline Total & 28 & 39,3139 & 2,38493 & \\
\hline Arquera & 4 & 10,2250 & 1,02770 & \\
\hline Defensa & 6 & 11,3800 & 0,97357 & \\
\hline Delantera & 6 & 11,1467 & 0,46315 & \multirow[t]{4}{*}{0,149} \\
\hline Medio defensiva & 6 & 11,3717 & 0,82137 & \\
\hline Medio ofensiva & 6 & 11,4733 & 0,60728 & \\
\hline Total & 28 & 11,1832 & 0,83508 & \\
\hline Arquera & 4 & 9,9200 & 0,71931 & \multirow{8}{*}{0,458} \\
\hline Defensa & 6 & 10,4600 & 0,95908 & \\
\hline Delantera & 6 & 10,0600 & 0,30312 & \\
\hline Medio defensiva & 6 & 10,6733 & 1,17651 & \\
\hline Medio ofensiva & 6 & 9,8483 & 0,92929 & \\
\hline Total & 28 & 10,2118 & 0,87705 & \\
\hline Arquera & 4 & 5,7250 & 0,41996 & \\
\hline Defensa & 6 & 6,0783 & 0,13934 & \\
\hline Delantera & 6 & 6,0900 & 0,48150 & \multirow[t]{6}{*}{0,648} \\
\hline Medio defensiva & 6 & 5,9817 & 0,39245 & \\
\hline Medio ofensiva & 6 & 6,0850 & 0,52622 & \\
\hline Total & 28 & 6,0111 & 0,40230 & \\
\hline Arquera & 4 & 3,9430 & 0,51352 & \\
\hline Defensa & 6 & 3,5466 & 0,34844 & \\
\hline Delantera & 6 & 3,5798 & 0,24900 & \multirow[t]{6}{*}{0,086} \\
\hline Medio defensiva & 6 & 3,4366 & 0,22601 & \\
\hline Medio ofensiva & 6 & 3,3115 & 0,34240 & \\
\hline Total & 28 & 3,5364 & 0,36444 & \\
\hline Arquera & 4 & 30,8755 & 4,51790 & \\
\hline Defensa & 6 & 27,7091 & 2,18536 & \\
\hline Delantera & 6 & 28,3973 & 3,16851 & \multirow[t]{4}{*}{0,629} \\
\hline Medio defensiva & 6 & 29,4330 & 3,01924 & \\
\hline Medio ofensiva & 6 & 29,9007 & 4,38760 & \\
\hline Total & 28 & 29,1480 & 3,38172 & \\
\hline
\end{tabular}


BAHAMONDES, A. C.; CIFUENTES, C. B. M.; LARA, P. E. \& BERRAL, R. F. J. Composición corporal y somatotipo en futbol femenino. Campeonato sudamericano sub-17. Int. J. Morphol., 30(2):450-460, 2012.

Tabla IV. Análisis estadístico comparativo por posición de juego: somatotipo.

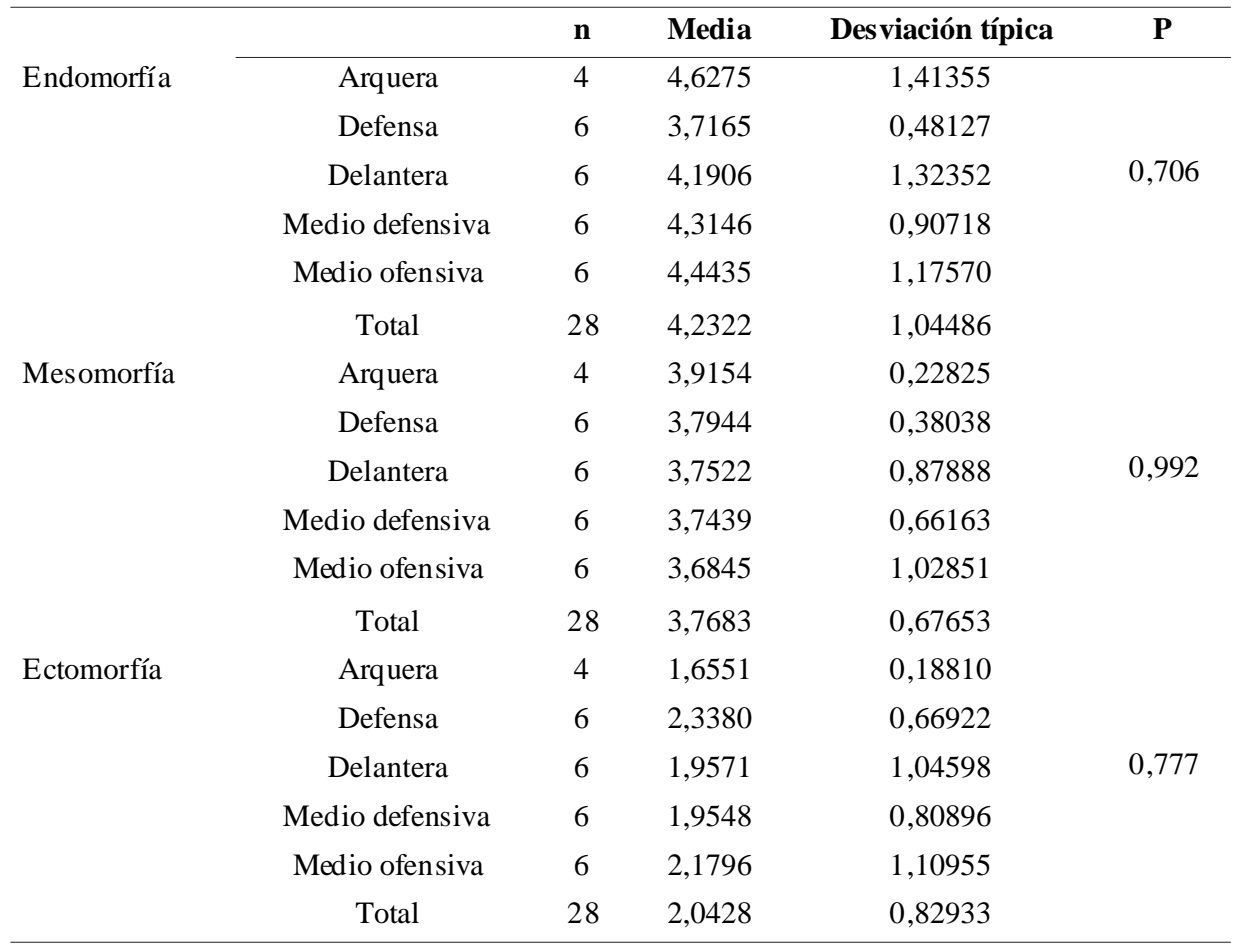

al., 2004), o de 25 seleccionadas chilenas Sub-20, con 18,1 $\pm 0,7$ años, donde presentaban un peso de $59,7 \pm 6,4 \mathrm{~kg}$ y una estatura de 159,2 \pm 5,0 cm (Almagià et al., 2008); o de 64 jugadoras universitarias americanas de 19,8 \pm 1,2 años, con un peso de $64,8 \pm 5,9 \mathrm{~kg}$ y una talla de $168,4 \pm 5,9 \mathrm{~cm}$ (Vescovi et al., 2006). La tendencia de encontrar arqueras más altas y pesadas en relación a los otros puestos de juego se presentó en este estudio, al igual que en otros, tanto en mujeres, como en hombres (Vescovi et al.; Queiroga et al., 2005; Rico-Sanz, 1998); en el caso de las defensoras, esto no está totalmente claro y difiere a lo citado por Rico-Sanz, pues aún siendo las mayores en edad, son las que pesan menos, ligado a una menor MA, comparado al resto; además se encuentran con una leve estatura sobre los otros grupos, a excepción de las arqueras. En cuanto a la ES y el IMC, Almagià et al. (2008), informan de valores de 83,6 6 2,1 $\mathrm{cm}$ y de $22,7 \pm 1,0 \mathrm{~kg} / \mathrm{mt}^{2}$, respectivamente, siendo menor a $\operatorname{los} 87,84 \pm 2,6 \mathrm{~cm}$ de ES y mayor a los $22,05 \pm 1,69 \mathrm{~kg} / \mathrm{mt}^{2} \mathrm{de}$ IMC de esta muestra (con ello, al relacionar en ambas muestras de evaluadas la E y ES por medio del Índice Córmico $\left(\mathrm{IC}=[\mathrm{ES} / \mathrm{E}]^{*} 100\right)$, se obtienen valores de $52,51 \%$ (Metricórmico) en la futbolistas sub 20 y un 54,76\% (Macrocórmico) (Betancourt \& Díaz Sánchez, 2007) en las sub 17, infiriendo una menor longitud relativa de extremidades inferiores en relación a su ES, dejando un posible y potencial aumento de su E total, a partir del desarrollo longitudinal de sus extremidades inferiores a medida que siga aumentando su edad; esta conducta es normal en muje- res de esa edad, incluso obteniendo valores de IC más elevados que varones de la misma edad (Rebato \& González, 1998); sin embargo, dadas las características del presente estudio no es posible objetivarlo, por lo demás no se debe olvidar que el crecimiento humano es alométrico, donde no todos los segmentos corporales tienen igual ritmo de crecimiento, (Betancourt \& Díaz Sánchez), y en el caso del IC, se ha documentado que puede ser afectado por unas piernas proporcionalmente más cortas, donde la longitud de estas ha sido considerada un indicador muy sensible de la exposición de las personas a las circunstancias socioeconómicas, condiciones de alimentación y el medio ambiente durante la infancia (Bronhara \& Ribeiro, 2007), instaurándose así un problema crónico de crecimiento, donde diferencias de estatura entre grupos de una misma población podría deberse a ello.

Los valores de $\sum 6 \mathrm{PL}$ (Tabla V) está por sobre lo informado en seleccionadas chilenas (Almagià et al., 2008) y bajo jugadoras de primera nacional españolas (Mujika et al.), también se presentan arriba de lo reportado en varias disciplinas deportivas femeninas, pero con menores valores en relación al promedio de un grupo de referencia de no deportistas (Norton \& Olds, 1996). Al compararlas con varones futbolistas de elite sudamericanos adultos, estas se encuentran por encima de los valores reportados para ellos (Rienzi et al., 1998). Otros autores han encontrado variaciones en los valores de IMC en tres grupos de 21,2 $\pm 3,1$ años, tras reali- 
BAHAMONDES, A. C.; CIFUENTES, C. B. M.; LARA, P. E. \& BERRAL, R. F. J. Composición corporal y somatotipo en futbol femenino. Campeonato sudamericano sub-17. Int. J. Morphol., 30(2):450-460, 2012.

Tabla V. Comparación de Masas Fraccionadas Plásticas con otros autores.

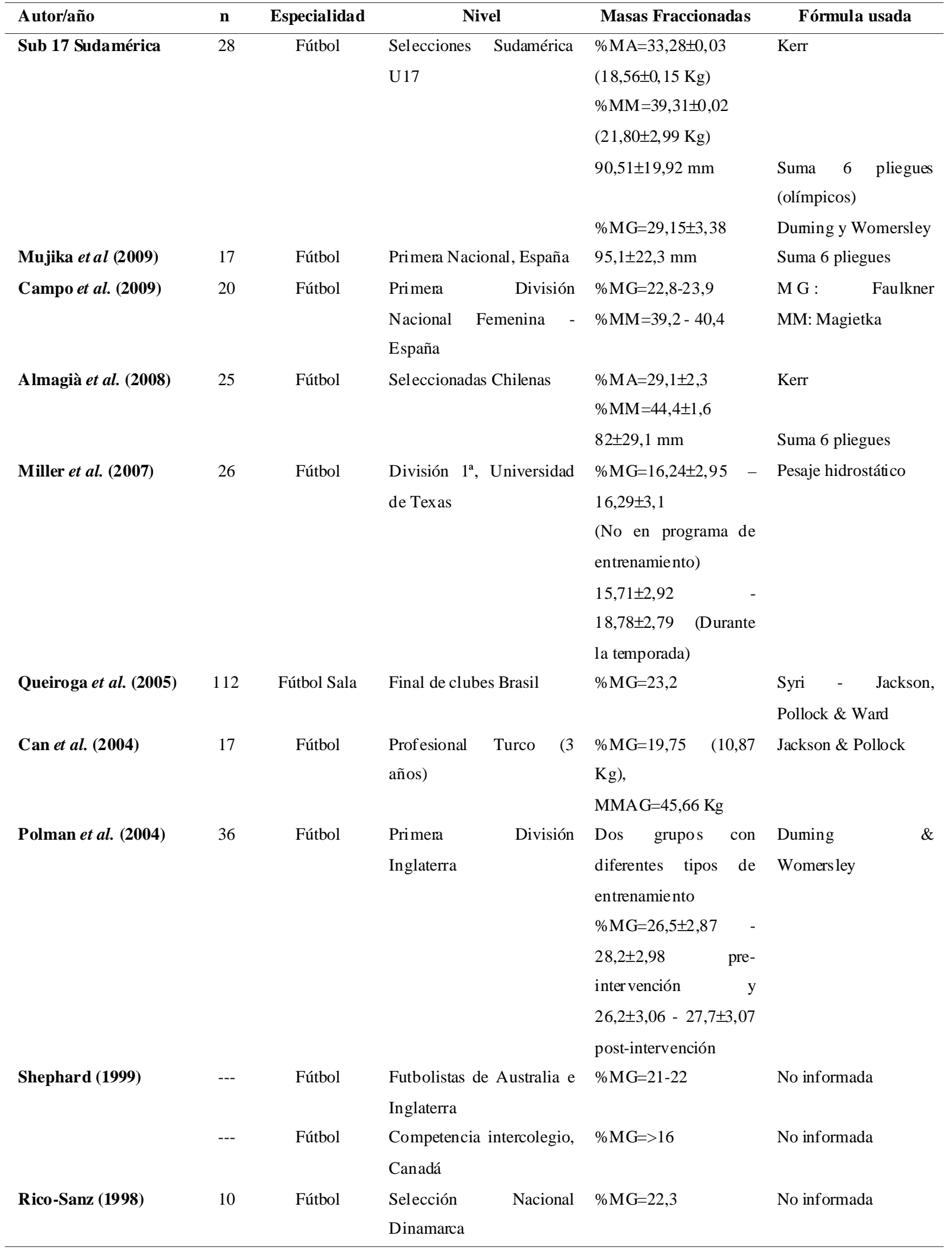

MA= Masa Adiposa - MG= Masa Grasa - MM= Masa Muscular - MMAG= Masa Magra. 
zar diferentes tipos de intervenciones, bajando desde 24,3 a $23,1 \mathrm{~kg} / \mathrm{mt}^{2}$ tras 12 semanas de entrenamiento (Polman et al., 2004), pero siendo estos valores mayores a los encontrados en este estudio. Una revisión realizada por Rico-Sanz en dos selecciones internacionales femeninas y un equipo universitario de 24,7; 25,4 y 20,3 años, señaló valores de IMC de 21,78; 21,62 y 21,90, respectivamente (Rico-Sanz), estando por debajo de los $22,05 \pm 1,69 \mathrm{~kg} / \mathrm{mt}^{2}$ del presente estudio.

En cuanto a las masas corporales, se han resumido diferentes metodologías de valoración en la Tabla V. Un estudio con seleccionadas chilenas de fútbol, pero no diferenciando puesto de juego, comunica que los \% de las masas son: MA de 29,1 \pm 2,3; la MM de 44,4 \pm 1,6; la MR de 10,3 \pm 0,9 y la MO de 10,6 \pm 0,8 (Almagià et al., 2008), siendo menores los valores de MM y MR, y más altos la MA y MO en las futbolistas Sub-17. Diversos estudios han evaluado la masa grasa (no adiposa) con algún modelo bicompartimental, de los cuales una publicación de la Universidad Metropolitana de Leeds indica que tras comparar a dos grupos de mujeres futbolistas inglesas de 21,2 $\pm 3,1$ años con diferentes tipos de entrenamiento, modificaron los valores de MG de 26,5 $\pm 2,87-28,2 \pm 2,98$ pre-intervención a 26,2 $\pm 3,06-27,7 \pm 3,07$ post-intervención (Polman et al.), los cuales estarían por debajo los 29,15 \pm 3,38 del presente estudio. En general, para modelos bicompartimentales antropométricos se han publicado datos en un rango de 16-23\% (Campo et al., 2009; Can et al.; Polman et al.; Queiroga et al., 2005; Rico-Sanz; Shephard, 1999; Vescovi et al.). El \% de MA para las jugadoras de fútbol femenino en este estudio fue superior a la media de seleccionadas chilenas y seleccionadas universitarias de fútbol y estudiantes de educación física chilenas, situación contraria al \% MM, donde están por debajo (Almagià et al., 2008; 2009). Estos resultados revelan que aunque el IMC de las participantes era el adecuado, gran parte de la masa corporal total era MA y no MM, situación que podría revertirse al combinar adecuadamente el entrenamiento y la nutrición (Polman et al.). Diversos estudios han demostrado que las mujeres deportistas, generalmente, tienen un 5-10\% más de grasa relativa que los hombres, aún así, es deseable competir con un cuerpo con baja grasa relativa, pues este tejido actúa como peso muerto, limitando la realización de acciones rápidas (Queiroga et al., 2008) y disminuyendo el rendimiento en aquellas actividades donde la masa corporal se mueve en el espacio, ya sea de forma vertical u horizontal (Can et al.). Ligeros aumentos de la muscularidad relativa, podrían ser beneficiosos para la producción de potencia en el futbol, así como en la prevención de lesiones (Vescovi et al.), a ello, Stolen et al. (2005), agregan que los equipos de fútbol menos cualificados en sus capacidades físicas tienen valores similares a los reportados hace 30 años. Sin embargo, dadas las características etarias de las evaluadas, no pueden excluirse los cambios de adiposidad propios de la pubertad y los años de entrenamiento como razones de las diferencias en las masas adiposa y muscular; se debe recordar que estas seleccionadas tuvieron su primera posibilidad de disputar un campeonato sudamericano clasificatorio a un mundial de fútbol, por lo que su experiencia deportiva de elite era incipiente y con poco tiempo de preparación, siendo esta relativa. Se debe recordar que los procesos de maduración no ocurren a la misma edad cronológica en todas las personas; así mujeres de una categoría, con la misma edad cronológica, pueden presentar diferencias en el rendimiento físico o motor, influyendo la capacidad funcional como jugadoras. Gutiérrez et al. (2010) relatan que, en general, en las categorías inferiores de futbol, los varones tienen ventaja si estos nacen en el primer semestre del año. Lo mismo acontece en el sexo femenino, lo que debería ser tomado en cuenta por los seleccionadores; en nuestro caso se cuenta con 16 y 12 jugadoras nacidas en cada semestre, durante los años 91, 92 y 93, respectivamente, no existiendo esa tendencia a concentrar jugadoras en el primer semestre; sin embargo, si se toman en cuenta solo las jugadoras mayores (año 91), la tendencia a concentrar es evidente, con 12 y 6 jugadoras nacidas en cada semestre, respectivamente. En el caso del IMO, se relaciona la cantidad de $\mathrm{kg}$ de MM con los $\mathrm{kg}$ de $\mathrm{MO}$, siendo un indicador de eficiencia mecánica, donde el músculo debe soportar o transportar una carga ósea, con ellos la biomecánica indica que la arquitectura ósea depende fundamentalmente de la sección transversal del músculo y del desarrollo de tensión que genere; se puede asociar con estados de salud (ej. atrofia muscular osteoporosis) o rendimiento deportivo (ej. hipertrofia), existiendo evidencia que puede llegar a valores cercanos a 5 en deportes como la Halterofilia. En este estudio el valor es de $3,5 \pm 0,36$, estando por debajo de los $3,7 \pm 0,4$ o los $3,9 \pm 0,4$ de jugadoras de Hockey de 22,6 \pm 3,3 años o Seleccionadas argentinas de 24,4 \pm 4,9 años, respectivamente, pero sobre los 3,4 \pm 0,4 de una población normal activa femenina de 26,1 $\pm 2,3$ años (Holway et al., 2009). Comparándolo con el estudio de futbolistas sudamericanos Sokip, y como es de esperar, los valores están por debajo de los reportados de 4,31 $\pm 0,11$ (Rienzi et al.).

Relativo al somatotipo, se han publicado valores en futbol femenino que se resumen en la Tabla VI, siendo consideradas endomorfas-mesomorfas (Can et al.); y endomesomorfas (Almagià et al., 2008); y en fútbol sala como meso-endomorfas (Queiroga et al., 2005; Levandosky et al.). Al comparar con futbolistas varones de la misma edad, se evidencia un predominio en estos de la mesomorfía sobre la endomorfía y ectomorfía (Reilly, 1997; Toteva, 2002). En este estudio no se demuestran diferencias de somatotipo de acuerdo a las posiciones de juego. Sin embargo, al comparar con otros estudios, se observa que los grupos evaluados son diferentes en su somatotipo, evidenciando biotipos diferentes (Tabla VI, Fig. 2); asumiendo que quizás a esta edad no es 
factor discriminante de rendimiento deportivo, donde la función de juego y el tiempo de entrenamiento no parecen causar modificaciones morfológicas en estas deportistas, o que el entrenamiento no haya sido realizado dentro del principio de la especificidad para cada posición (Queiroga et al., 2005).

Es evidente que la participación femenina en el fútbol continuará en el futuro, por lo que son necesarias más investigaciones. Se sugiere considerar la función dentro del campo de juego a la hora de interpretar la composición corporal (Carling \& Orhant, 2010), así como describir el nivel de rendimiento u otros factores determinantes del entrenamiento (Can et al.). Se espera que la información obtenida sea útil para vislumbrar resultados deportivos de futuras selecciones y contribuir en la mejora de los criterios de selección de talentos en el fútbol.

Tabla VI. Comparación de Somatotipo con otros autores: somatotipo medio, puntos de la somatocarta y distancia de dispersión del somatotipo medio (SDD del SM).

\begin{tabular}{|c|c|c|c|c|c|c|c|c|c|}
\hline Autor/Año & $n$ & Especialidad & Nivel & ENDO & MESO & ECTO & $X$ & $Y$ & $\begin{array}{c}S D D d e l \\
S M\end{array}$ \\
\hline Sub 17 Sudamérica & 28 & Fútbol & $\begin{array}{c}\text { Selecciones } \\
\text { Sudamérica } \\
\text { U17 }\end{array}$ & 4,23 & 3,77 & 2,04 & $-2,2$ & 1,3 & \\
\hline Almagià et al. (2008 & 25 & Fútbol & $\begin{array}{c}\text { Selección } \\
\text { Chilena U20 }\end{array}$ & 3,90 & 4,70 & 1,50 & $-2,40$ & 4,00 & 2,8 \\
\hline $\begin{array}{l}\text { Levandosky et al. } \\
(2007)\end{array}$ & 15 & Fútbol sala & $\begin{array}{c}\text { Juegos } \\
\text { Paraná - } \\
\text { Brasil }\end{array}$ & 5,37 & 3,34 & 2,06 & $-3,31$ & $-0,75$ & 2,8 \\
\hline Queiroga et al. (2005) & 112 & Fútbol Sala & $\begin{array}{c}\text { Final de } \\
\text { clubes Brasil }\end{array}$ & 5,00 & 3,30 & 2,10 & $-2,90$ & $-0,50$ & 2,1 \\
\hline Can et al. (2004) & 17 & Fútbol & $\begin{array}{c}\text { Profesional } \\
\text { Turco (3 } \\
\text { años) }\end{array}$ & 3,07 & 3,55 & 2,43 & $-0,64$ & 1,60 & 2,7 \\
\hline
\end{tabular}

Fig. 2. Comparación de somatotipos medios con otros autores.

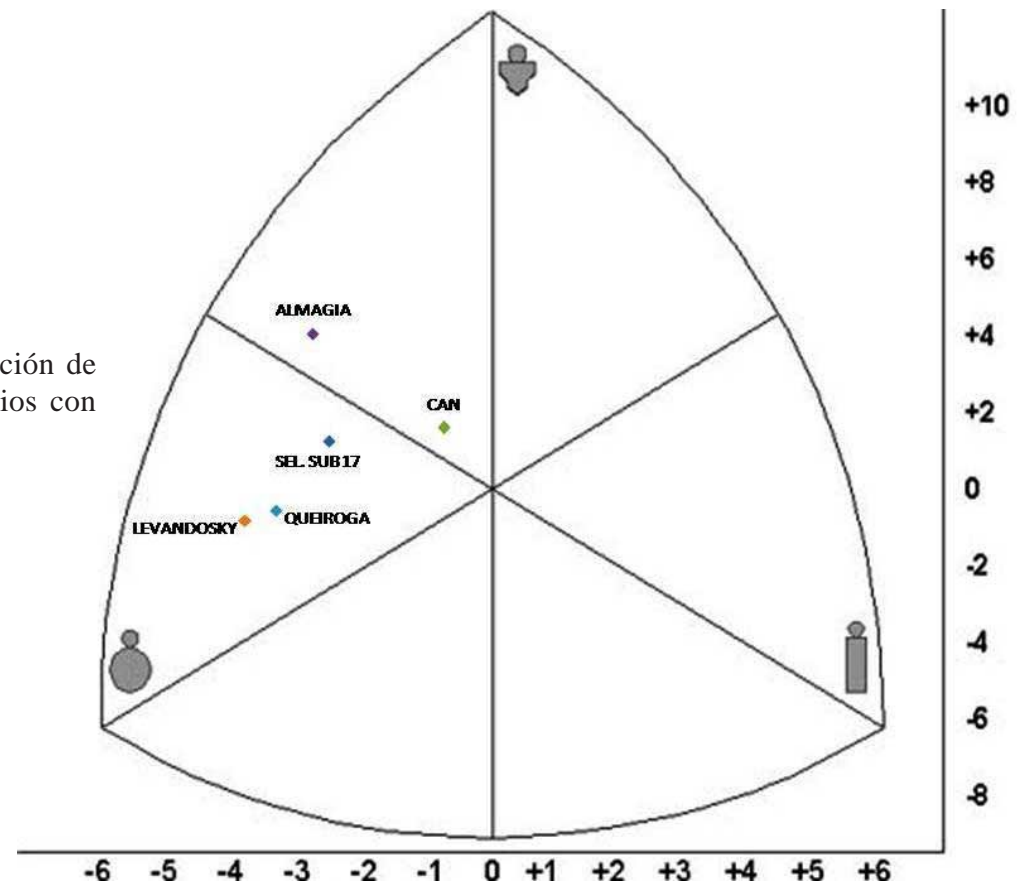




\section{AGRADECIMIENTOS}

A la Dirección Regional de la Universidad Mayor, sede Temuco, por aportar en el financiamiento de la investigación. A los cuerpos técnicos y médicos de ambas selecciones por facilitar el acceso y autorizar el evaluar a sus deportistas.

BAHAMONDES, A. C.; CIFUENTES, C. B. M.; LARA, P. E. \& BERRAL, R. F. J. Body composition and somatotype in women's football. South american championship sub-17. Int. J. Morphol., 30(2):450-460, 2012.

SUMMARY: In order to analyze body composition and somatotype in women selected to participate in the national soccer teams, we studied 28 women, 19 selected from Columbia and 9 from Paraguay, with an average of $16.2 \pm 0.66$ years of age, participating in the South American Championship Sub 17 - Chile 2008, a qualifying event for the FIFA World Cup first category, New Zealand 2008. The evaluation followed the protocol established by the International Society for the Advancement of Kinanthropometry - ISAK. We determined the classification of total body mass in five types according to Kerr. To establish the somatotype the model proposed by Heath \& Carter was used. Regarding body mass, the average $\mathrm{kg}$ and $\%$ of MA, MM and MO was $18.56 \mathrm{~kg}-33.28 \% ; 21.80 \mathrm{~kg}-39.31 \%$; $6.20 \mathrm{~kg}-11.18 \%$. The classification of somatotype was endomorph-mesomorph, where there is an equal dominance of adiposity and muscularity on the relative linearity. The SDD of SM indicates no significant differences $(p<0.05)$ exists among the different positions of the players, and the SDI suggests that the total group of evaluated players is homogeneous.

KEY WORDS: Soccer; Anthropometry; Muscle performance; Muscle-bone ratio; Biotype.

\section{REFERENCIAS BIBLIOGRAFICAS}

Almagià, A. A.; Rodriguez, F. J.; Barraza, F. O.; Lizana, P. J. \& Jorquera, C. A. Perfil antropométrico de jugadoras chilenas de futbol femenino. Int. J.Morphol., 26(4):817-21, 2008.

Almagià, A. A.; Lizana, P. J.; Rodríguez, F. J.; Ivanovic, M. D. \& Binvignat, G. O. Variables antropométricas y rendimiento físico en estudiantes universitarios de educación física. Int. J. Morphol., 27(4):971-5, 2009.

Berral, F. J.; Rodríguez-Bies, E.; Berral, C.; Rojano, D. \& Lara, E. Comparación de Ecuaciones Antropométricas para Evaluar la Masa Muscular en Jugadores de Badminton. Int. J. Morphol., 28(3):803-10, 2010.
Betancourt, H. \& Díaz Sánchez, M. E. Estimación de las relaciones de proporcionalidad de adolescentes bailarines de ballet. Rev. Int. Med. Cienc. Act. Fís. Deporte, 7(28):330-43, 2007.

Bronhara, B. \& Ribeiro, V. C. Body proportion in anthropometric assessment of post-menarche adolescents. Rev. Nutr., 20(1):2737, 2007.

Campo, S. S.; Vaeyens, R.; Philippaerts, R. M.; Redondo, J. C.; De Benito, A. M. \& Cuadrado, G. Effects of lower-limb plyometric training on body composition, explosive strength and kicking speed in female soccer players. J. Strength Cond. Res., 23(6):1714-22, 2009.

Can, E.; Yilmaz, I. \& Erden, Z. Morphological characteristics and performance variables of women soccer players. J. Strength Cond. Res., 18(3):480-5, 2004.

Carling, C. \& Orhant, E. Variation in Body Composition in Professional Soccer Players: Interseasonal and Intraseasonal Changes and the Effects of Exposure Time and Player Position. J. Strength Cond. Res., 24(5):1332-9, 2010.

Carter, J. E. L. \& Heath, B. H. Somatotyping: development and applications. Cambridge, Cambridge University Press, 1990.

Carter, J. E. L. Somatotipo. In: Antropométrica. $1^{\mathrm{a}}$ ed. Buenos Aires, Biosystem Servicio Educativo, 2000.

Esparza Ros, F. Manual de Cineantropometría. $1^{\mathrm{a}}$ ed. Madrid, Femede, 1993

Gutierrez, D.; Pastor, J. C.; González, S. \& Contreras, O. The relative age effect in youth soccer players from Spain. JSSM, 9:190-8, 2010.

Heath, B. H. \& Carter, J. E. L. A modified somatotype method. Am. J. Phys. Anthrop., 27:57-74, 1967.

Holway, F.; Miguez, J.; Pudelka, M. \& Pastor, M. Características morfológicas de jugadoras de hockey de elite argentinas. Rev. Electrón. Cienc. Apl. Deporte, 2(6):2009. Disponible en: http:// www.romerobrest.edu.ar/ojs/index.php/ReCAD/article/view/37

Kerr, D. A. An anthropometric method for fractionation of skin, adipose, bone, muscle and residual masses in males and females age 6 to 77 years. M. Cs. Kinesiology Tesis. British Columbia, Simon Fraser University, 1988.

Levandosky, G.; Cardozo, F. L.; Cieslak, F. \& Cardoso, A. S. Somatotype profile, anthropometric variables, physical aptitude and motor behavior of juvenile athletes of female futsal time from Ponta Grossa (Paraná-Brazil). Fit. Perf. J., 6(3):162-6, 2007.

Marfell-Jones, M.; Olds, T.; Stewart, A. D. \& Carter, L. International Standards for Anthropometric Assessment. Potchefstroom, South Africa, International Society for the Advancement of Kinanthropometry (ISAK), 2006. pp.61-75. 
Miller, T.; Thierry-Aguilera, R.; Congleton, J.; Amendola, A.; Clark, M.; Crouse, S.; Martin, S. \& Jenkins, O. Seasonal Changes In Vo2max Among Division 1a Collegiate Women Soccer Players. J. Strength Cond. Res., 21(1):48-51, 2007.

Mujika, I.; Santisteban, J.; Impellizzeri, F. \& Castagna, C. Fitness determinants of success in men's and women's football. $J$. Sports Sci., 27(2):107-14, 2009.

Norton, K. \& Olds, T. Antropométrica. Marrickville, Sidney, Southwood Press, 1996.

Polman, R.; Walsh, D.; Bloomfield, J. \& Nesti, M. Effective conditioning of female soccer players. J. Sport Sci., 22:191203, 2004.

Queiroga, M.; Ferreira, S. \& Romanzini, M. Perfil antropométrico de atletas de futsal feminino de alto nível competitivo conforme a funçao tática desempanhada no jogo. Rev. Bras. Cine. Des. Hum., 7(1):30-4, 2005.

Queiroga, M.; Ferreira, S.; Pereira, G. \& Kokubun, E. Somatotipo como indicador de desempenho em atletas de futsal feminino. Rev. Bras. Cine. Des. Hum., 10(1):56-61, 2008.

Rebato, E. \& González, A. Evaluación antropométrica del crecimiento somático en adolescentes del medio urbano. Zainak: Cuadernos de Antropología-Etnografía, 16:7-22, 1998.

Reilly, T. Perfil fisiológico del jugador de fútbol. Proceedings V Simposio Internacional. Actualización en Ciencias Aplicadas al Deporte, Edit. Biosystem, 1997. pp.402-16, Cap. 3.

Reilly, T.; Bangsbo, J. \& Franks, A. Anthropometric and physiological predisposition for elite soccer. J. Sport Sci., 18:669-83, 2000.

Rico-Sanz, J. Body composition and nutritional assessments in soccer. Int. J. Sports Nutr., 8:113-23, 1998.

Rienzi, E.; Mazza, J. C.; Carter, J. E. L. \& Reilly, T. SOKIP, Soccer Kinanthropometric Project I. Futbolista sudamericano de élite: Morfología, análisis del juego y performance. Rosario, Editorial Biosystem Servicio Educativo, 1998.

Sharma, S. S. \& Dixit, N. K. Somatotype of athletes and their performance. Int. J. Sports Med., 6:161-2, 1985.

Shephard, R. J. Biology and medicine of soccer: An update. J. Sports Sci., 17(10):757-86. 1999.

Stolen, T.; Chamari, K.; Castagna, C. \& Wisloff, U. Physiology of soccer: an update. Sports Med., 35(6):501-36, 2005.

Toteva, M. Somatotype characteristicsof young football players. In: Spinks, W. \& Reilly, T. Science and Football IV. Proceedings of the 4th World Congress of Science and Football. Nueva York, Routledge, 2002. pp.263-4.
Vescovi, J. D.; Brown, T. D. \& Murray, T. M. Positional characteristics of physical performance in Division I college female soccer players. J. Sports Med. Phys. Fitness, 46(2):2216, 2006.

\section{Dirección para correspondencia: \\ Carlos Bahamondes Avila.}

Laboratorio de Fisiología y Movimiento Humano

Carrera de Kinesiología

Universidad Mayor - Sede Temuco

CHILE

\section{Email: carlos.bahamondesa@mayor.cl fjberde@upo.es}

Recibido : 21-11-2011

Aceptado:22-03-2012 